\title{
IMPROVED RESULTS ON THE NO-BINDING OF BIPOLARONS
}

\author{
RAFAEL D. BENGURIA ${ }^{1}$ AND GONZALO A. BLEY ${ }^{2}$
}

\begin{abstract}
For the case of the bipolaron, it has been recently proved that for $U \geq 53.2 \alpha$, where $U$ is the repulsion parameter of the electrons, and $\alpha$ is the coupling constant of the polaron, no binding occurs. We show that actually for $U \geq 52.1 \alpha$, there is no binding. Furthermore, we obtain special results for small and large values of $\alpha$ : more specifically, we prove that for each $\varepsilon>0$, there is an $\alpha_{1}$ and an $\alpha_{2}$, such that if $0<\alpha \leq \alpha_{1}$, a condition for no-binding becomes $U \geq(40.4+\varepsilon) \alpha$, and if $\alpha \geq \alpha_{2}$, it is $U \geq(38.7+\varepsilon) \alpha$. Here, $\alpha_{1}$ can be computed with any desired accuracy, whereas we were only able to prove the existence of such an $\alpha_{2}$.
\end{abstract}

\section{INTRODUCTION}

The polaron was first considered by Fröhlich [9, 10] as a model of one non-relativistic electron interacting with the longitudinal, optical phonons in the periodic lattice of atoms of a polar crystal (e.g., $\mathrm{NaCl}$ ). More precisely, the polaron is a quasi-particle, which corresponds to the electron plus the phonons created by the interaction of the electron with the electric field of the neighboring ions. Fröhlich derived the Hamiltonian of the polaron with the immediate interest of explaining the phenomenon of electrical breakdown in polar crystals. It was later called the "large" polaron, since in the formulation of the model, it was assumed that the distortion of the lattice, due to the electron, comprised several lattice constants, and so the medium was treated as a continuum. Since the creation of the model and its corresponding Hamiltonian, the study of polarons has been in part stimulated by the fact that it is a simple description of a particle interacting with a quantum field, in the context of non-relativistic quantum mechanics. For a self-contained introduction to polarons, the reader may consult [19]. Also, a very complete review of what was known about it, until 1969, is in [3].

The $\mathrm{N}$-polaron is the extension to the case of $\mathrm{N}$ electrons, interacting not only with the field of phonons, but also with each other through their mutual Coulomb repulsion. N-polarons may play an important role in superconductivity at high temperature [1, 2], so there is some interest in knowing under what circumstances they may be formed, that is, the groundstate energy of the $\mathrm{N}$-polaron is smaller than $\mathrm{N}$ times that of the polaron (and thus the $\mathrm{N}$-polaron configuration being energetically more favorable). In studying this question, we will concentrate ourselves only on the bipolaron.

1.1. The bipolaron Hamiltonian. We first introduce the Hamiltonian of the polaron,

$$
H^{1}=p^{2}-\frac{1}{\pi} \sqrt{\frac{\alpha}{2}} \int\left[a(k) e^{i k x}+a(k)^{\dagger} e^{-i k x}\right] \frac{d k}{|k|}+\int a(k)^{\dagger} a(k) d k .
$$

R. B. was supported in part by the Iniciativa Científica Milenio, ICM (CHILE) project P07-027-F, and by FONDECYT (Chile) Project 1100679.

G. B. was supported in part by the Iniciativa Científica Milenio, ICM (CHILE) project P07-027-F. 
As it is usual, whenever we omit the subscript in the integrals, integration in $\mathbb{R}^{3}$ is understood. Moreover, $x \in \mathbb{R}^{3}$ is the position of the electron, and $p$ is the corresponding momentum. Here, $a(k)$ denotes the lowering operator for the harmonic oscillator corresponding to the mode $k \in \mathbb{R}^{3}$ of the field, and this operator and its adjoint satisfy the canonical commutation relation $\left[a(k), a\left(k^{\prime}\right)^{\dagger}\right]=\delta\left(k-k^{\prime}\right)$, where $\delta$ is the Dirac delta. The first term corresponds to the kinetic energy of the electron ( $p$ is given by $-i \nabla)$, the second one to the interaction of the electron with the phonon field, and the last term is the energy of this field. The units used here are such that $\hbar=2 m=\omega=1$, where $m$ is the band mass of the electron, and $\omega$ is the frequency of the phonons. We point out the fact that the dispersion relation is indeed $\omega=1$, that is, there is no dependence of the frequency of the phonons upon the mode of the field; this is part of the assumptions made for the derivation of this Hamiltonian by Fröhlich. This operator will act on the Hilbert space $L^{2}\left(\mathbb{R}^{3}\right) \otimes \mathcal{F}$, where $\mathcal{F}$ is the Fock space associated to the phonons. Here, $\alpha$ is a positive, dimensionless constant, known as the coupling constant for the polaron, and there is an explicit expression for it; restoring units, it is given by,

$$
\alpha=\frac{e^{2}}{2 \hbar \omega}\left(\frac{2 m \omega}{\hbar}\right)^{1 / 2}\left(\frac{1}{\epsilon_{\infty}}-\frac{1}{\epsilon_{0}}\right),
$$

where $\omega$ is as before, the frequency of the phonons, $e$ is the charge of the electron, and $\epsilon_{0}$ and $\epsilon_{\infty}$ are known as the static and high-frequency dielectric constants of the material, respectively.

The bipolaron Hamiltonian is simply a generalization of the previous operator, and is obtained by including the electronic repulsion:

$$
\begin{aligned}
H_{U}^{2}= & p_{1}^{2}+p_{2}^{2}-\frac{1}{\pi} \sqrt{\frac{\alpha}{2}} \int\left[a(k) e^{i k x_{1}}+a(k)^{\dagger} e^{-i k x_{1}}\right] \frac{d k}{|k|} \\
& -\frac{1}{\pi} \sqrt{\frac{\alpha}{2}} \int\left[a(k) e^{i k x_{2}}+a(k)^{\dagger} e^{-i k x_{2}}\right] \frac{d k}{|k|}+\int a(k)^{\dagger} a(k) d k \\
& +\frac{U}{\left|x_{2}-x_{1}\right|},
\end{aligned}
$$

where $x_{i}$ and $p_{i}$ are the position and the momentum of the $i$-th particle. Here, $U$ is, in these units, a dimensionless parameter. It is given by $e^{2} / \epsilon_{\infty}$, and it satisfies an important relation: notice that,

$$
\alpha<\frac{e^{2}}{2 \hbar \omega}\left(\frac{2 m \omega}{\hbar}\right)^{1 / 2} \frac{1}{\epsilon_{\infty}},
$$

and so we must have $U>2 \alpha$. We will assume this condition hereafter.

Given $N$, the number of electrons, we are interested in the ground-state energy of $H_{U}^{N}(N$ being 1 or 2 , and $H_{U}^{1}$ being written as $H^{1}$ ), denoted by $E_{U}^{N}$, which is defined by,

$$
E_{U}^{N}=\inf \left\{\left\langle\psi\left|H_{U}^{N}\right| \psi\right\rangle: \psi \in L^{2}\left(\mathbb{R}^{3 N}\right) \otimes \mathcal{F},\|\psi\|=1\right\}
$$

As can be seen from the definition of $E_{U}^{N}$, we will not treat the electrons as fermions, spin will also be discarded. $E_{U}^{1}$ will be denoted by $E^{1}$. Finally, the binding energy of the bipolaron is given by,

$$
2 E^{1}-E_{U}^{2} .
$$


1.2. Bipolaron formation. With the information at hand, we can now state the question presented previously in more precise terms: given $\alpha>0$, for what values of $U$ will bipolarons not exist?, that is, for what values of $U, E_{U}^{2}=2 E^{1}$, and so making the binding energy equal to zero? It will suffice to find values of $U$ for which $E_{U}^{2} \geq 2 E^{1}$ because the other inequality is always true, due to the subadditivity of the energy, $E_{U}^{2} \leq 2 E^{1}[11$, 12, 16] which is valid in a more general setting.

In a recent article, Frank, Lieb, Seiringer and Thomas [7, 8] have proved that, for any $\alpha>0$, no binding occurs if $U \geq 53.2 \alpha$, thus finding an interesting linear law. In particular, it shows that bipolarons will not be formed if the mutual repulsion among the electrons is sufficiently strong; in this case, the attraction that is caused by the electric field of the ions is not able to overcome this repulsion. It is known that bipolarons will be formed if $U \leq 2.3 \alpha$, at least in the limit $\alpha \rightarrow \infty$ [20, 21], and in this case the opposite phenomenon occurs: the electric field due to the ions of the lattice is able to keep the electrons bound together.

Assuming that the bipolaron Hamiltonian is bounded from below (which will become apparent soon), it is seen that $E_{U}^{2}$ is a monotone increasing function of $U$, which arises from taking an inner product with some vector in the Hilbert space, and then bounding appropriately and obtaining the infimum at both sides. Thus, the result of no binding for large enough $U$ would be deduced if we knew that no binding occurs for some $U$.

Whether or not the correct behavior is a linear law (recall that if $U \geq 53.2 \alpha$, no binding occurs), we may generalize by stating that there is a function $U_{c}(\alpha)$, such that no bipolaron will be formed if $U>U_{c}(\alpha)$, and binding will occur if $U<U_{c}(\alpha)$. This function exists since we can actually define it as,

$$
U_{c}(\alpha)=\inf \left\{U>2 \alpha: 2 E^{1}(\alpha)=E_{U}^{2}(\alpha)\right\} .
$$

A priori, the infimum may belong to the set or not, so we do not mention the case $U=U_{c}(\alpha)$. This function could be $2 \alpha$, for some $\alpha$; actually, some works suggest that $\lim _{\alpha \rightarrow 0^{+}} U_{c}(\alpha) / 2 \alpha=1$ [4, 21, 22], however we believe no formal proof of this result exists. On the other hand, we do not know how small the value,

$$
\sup _{\alpha>0} \frac{U_{c}(\alpha)}{\alpha}
$$

could be. The previously stated result affirms that it is smaller than or equal to 53.2.

\section{Overview of the Results}

We will now explain each of the results obtained; the proofs will be presented in the next sections:

a) An improvement upon $\sup _{\alpha>0} U_{c}(\alpha) / \alpha$. We were able to improve slightly the numerical constant 53.2 to 52.1 , which corresponds to a reduction of about two percent. The proof is based on a method developed in [7]. (See Section 3 below).

b) The case of weak coupling $\alpha \rightarrow 0$. For sufficiently small coupling constant $\alpha$, the previous result has been lowered; though the relation is no longer linear. More specifically, we have found an explicit error function $\delta_{1}(\alpha)$, such that for any $\alpha<3 / 4$, if,

$$
U \geq 40.4 \alpha+\delta_{1}(\alpha)
$$

where $\delta_{1}(\alpha)>0$, and $\lim _{\alpha \rightarrow 0} \delta_{1}(\alpha) / \alpha=0$, then $E_{U}^{2}(\alpha)=2 E^{1}(\alpha)$. By definition of limit, this can be stated in a different form: for each $\varepsilon>0$, there is a $\alpha_{1}>0$, such that if $0<\alpha \leq \alpha_{1}$, 
no binding occurs provided $U \geq(40.4+\varepsilon) \alpha$ (and thus recovering a linear law). This $\alpha_{1}$ is unique and can be computed with any desired accuracy, as we shall see below. (See Section 4 below).

c) The case of strong coupling $\alpha \rightarrow \infty$ We have obtained an analogous result in the limit $\alpha \rightarrow \infty$. An error function $\delta_{2}(\alpha)>0$ is found, such that, for all $\alpha>0$, if,

$$
U \geq 38.7 \alpha+\delta_{2}(\alpha)
$$

where $\delta_{2}(\alpha) / \alpha \rightarrow 0$ as $\alpha \rightarrow \infty$, then no binding occurs. Alluding again to the definition of limit, this result can be cast in a similar form to that shown in the case of weak coupling. However, contrary to that situation, we are only able to prove that a corresponding value exists, not to evaluate it. (See Section 5 below).

\section{AN IMPROVED LINEAR LAW}

Here we prove the following theorem,

Theorem 3.1. For any $\alpha>0$, if $U \geq 52.1 \alpha$, no bipolaron is formed.

As was said previously, the proof is based on a method used in [7]. Actually, it is almost the same, except that we consider the variables that appear there in a more general setting. We begin by performing a partition of unity [5, Section 3.1] on the variable $\left|x_{2}-x_{1}\right|$, the relative distance between the two electrons. To this end, consider the family of functions $\varphi_{j}$, given by,

$$
\begin{gathered}
\varphi_{1}(t)= \begin{cases}\cos \left(\frac{\pi t}{2 a_{1}}\right) & 0 \leq t<a_{1} \\
0 & t \geq a_{1},\end{cases} \\
\varphi_{2}(t)= \begin{cases}\sin \left(\frac{\pi t}{2 a_{1}}\right) & 0 \leq t<a_{1} \\
\cos \left[\frac{\pi\left(t-a_{1}\right)}{2 a_{2}}\right] & a_{1} \leq t<a_{1}+a_{2} \\
0 & t>a_{1}+a_{2},\end{cases} \\
\varphi_{j}(t)= \begin{cases}\sin \left[\frac{\pi\left(t-S_{j-2}\right)}{2 a_{j-1}}\right] & S_{j-2} \leq t \leq S_{j-1} \\
\cos \left[\frac{\pi\left(t-S_{j-1}\right)}{2 a_{j}}\right] & S_{j-1} \leq t \leq S_{j} \\
0 & \text { otherwise, }\end{cases}
\end{gathered}
$$

where $S_{j}=\sum_{i=1}^{j} a_{i}$. For the moment, $a_{j}$ are arbitrary numbers; we only impose that $a_{j}>0$ and $\sum_{i=1}^{\infty} a_{i}=\infty$. Equation (13) is written for $j \geq 3$. We see that for any $t \in[0, \infty)$,

$$
\sum_{i=1}^{\infty} \varphi_{i}(t)^{2}=1
$$


Now, we partition $\left|x_{2}-x_{1}\right|$ with this set of functions, and (using the standard IMS localization formula, see, e.g., [5]) we conclude that,

$$
\begin{aligned}
H_{U}^{2} & =\sum_{i=1}^{\infty} \varphi_{i}\left(\left|x_{2}-x_{1}\right|\right) H_{U}^{2} \varphi_{i}\left(\left|x_{2}-x_{1}\right|\right)-2 \sum_{i=1}^{\infty} \varphi_{i}^{\prime}\left(\left|x_{2}-x_{1}\right|\right)^{2} \\
& =\sum_{i=1}^{\infty} \varphi_{i}\left[H_{U}^{2}-R\right] \varphi_{i}
\end{aligned}
$$

where,

$$
R \equiv 2 \sum_{i=1}^{\infty} \varphi_{i}^{\prime}\left(\left|x_{2}-x_{1}\right|\right)^{2}
$$

is the localization error.

Given $\psi \in L^{2}\left(\mathbb{R}^{6}\right) \otimes \mathcal{F}$, with $\|\psi\|=1$, we can write $\left\langle\psi\left|H_{U}^{2}\right| \psi\right\rangle$ as,

$$
\sum_{i=1}^{\infty}\left\langle\psi \varphi_{i}\left|\left(H_{0}^{2}+\frac{U}{\left|x_{2}-x_{1}\right|}-R\right)\right| \psi \varphi_{i}\right\rangle
$$

If we are able to find a $U_{0}(\alpha)$, such that for any $U \geq U_{0}(\alpha)$,

$$
\left\langle\psi \varphi_{j}\left|\left(H_{0}^{2}+\frac{U}{\left|x_{2}-x_{1}\right|}-R\right)\right| \psi \varphi_{j}\right\rangle \geq 2 E^{1}\left\|\psi \varphi_{j}\right\|^{2}
$$

for each $j \geq 1$, then,

$$
\left\langle\psi\left|H_{U}^{2}\right| \psi\right\rangle \geq 2 E^{1}
$$

from which the conclusion will follow. For $j=1$, we will have,

$$
\left\langle\psi \varphi_{1}\left|\left(H_{0}^{2}+\frac{U}{\left|x_{2}-x_{1}\right|}-R\right)\right| \psi \varphi_{1}\right\rangle \geq\left\langle\psi \varphi_{1}\left|\left(H_{0}^{2}+\frac{U}{a_{1}}-\frac{\pi^{2}}{2 a_{1}^{2}}\right)\right| \psi \varphi_{1}\right\rangle,
$$

and now we use Lemma 2 of reference [7]; for us it will be called Lemma 1:

Lemma 3.2. For all $\alpha>0$, if $\phi \in L^{2}\left(\mathbb{R}^{6}\right) \otimes \mathcal{F}$,

$$
\left\langle\phi\left|H_{0}^{2}\right| \phi\right\rangle \geq\left[2 E^{1}-\frac{7}{3} \alpha^{2}\right]\|\phi\|^{2} .
$$

Therefore, the right hand side of (20) is bounded from below by,

$$
\left[2 E^{1}+\frac{U}{a_{1}}-\frac{\pi^{2}}{2 a_{1}^{2}}-\frac{7}{3} \alpha^{2}\right]\left\|\psi \varphi_{1}\right\|^{2}
$$

Then the condition (18) will be satisfied if $U$ is such that,

$$
U \geq \frac{7}{3} \alpha^{2} a_{1}+\frac{\pi^{2}}{2 a_{1}} .
$$

For $j=2$, we repeat the argument, and find that it is enough for $U$ to satisfy,

$$
U \geq\left[\frac{7}{3} \alpha^{2}+\frac{\pi^{2}}{2 \min \left(a_{1}, a_{2}\right)^{2}}\right]\left(a_{1}+a_{2}\right),
$$

where min denotes the minimum of its two arguments. 
Since for any $a_{1}, a_{2}>0$,

$$
\left[\frac{7}{3} \alpha^{2}+\frac{\pi^{2}}{2 \min \left(a_{1}, a_{2}\right)^{2}}\right]\left(a_{1}+a_{2}\right) \geq\left(\frac{7}{3} \alpha^{2}+\frac{\pi^{2}}{2 a_{1}^{2}}\right)\left(a_{1}+a_{2}\right) \geq \frac{7}{3} \alpha^{2} a_{1}+\frac{\pi^{2}}{2 a_{1}},
$$

it will suffice if the second condition $(j=2)$ is satisfied.

For $j \geq 3$, we have the following lower bound for the left hand side of $(18)$ :

$$
\left\langle\psi \varphi_{j}\left|H_{0}^{2}\right| \psi \varphi_{j}\right\rangle+\left\langle\psi \varphi_{j}\left|\frac{U}{\left|x_{2}-x_{1}\right|}\right| \psi \varphi_{j}\right\rangle-\frac{\pi^{2}}{2 \min \left(a_{j-1}, a_{j}\right)^{2}}\left\|\psi \varphi_{j}\right\|^{2},
$$

and considering the operator $H_{0}^{2}$, we perform a partition of $\mathbb{R}^{6}$ consisting of spheres, one for each particle: we take the family of functions,

$$
g_{j, u_{1}, u_{2}}\left(x_{1}, x_{2}\right)=\frac{1}{L_{j}^{3}} \chi\left(\frac{x_{1}-u_{1}}{L_{j}}\right) \chi\left(\frac{x_{2}-u_{2}}{L_{j}}\right),
$$

indexed by $j$ and $u_{1}, u_{2} \in \mathbb{R}^{3}$, where $L_{j}>0$ will be the radius of each ball, and $\chi$ is given by,

$$
\chi(x)=\frac{1}{\sqrt{2 \pi}|x|} \begin{cases}\sin (\pi|x|) & |x| \leq 1 \\ 0 & |x|>1\end{cases}
$$

and is such that $\int \chi^{2} d x=1$. Then,

$$
\iint g_{j, u_{1}, u_{2}}\left(x_{1}, x_{2}\right)^{2} d u_{1} d u_{2}=1
$$

and so a similar localization formula can be obtained, by using the same arguments used to derive the more usual one involving sums [5, Section 3.1]. Hence,

$$
H_{0}^{2}=\iint g_{j, u_{1}, u_{2}}\left(x_{1}, x_{2}\right) H_{0}^{2} g_{j, u_{1}, u_{2}}\left(x_{1}, x_{2}\right) d u_{1} d u_{2}-\frac{2 \pi^{2}}{L_{j}^{2}} .
$$

We have used the fact that $\int|\nabla \chi|^{2} d x=\pi^{2}$, which follows from an integration by parts. Also, $\chi$ is the function that minimizes the localization error. We can now write 26 as,

$$
\begin{gathered}
\iint\left\langle\psi \varphi_{j} g_{j, u_{1}, u_{2}}\left|H_{0}^{2}\right| \psi \varphi_{j} g_{j, u_{1}, u_{2}}\right\rangle d u_{1} d u_{2}+ \\
\left\langle\psi \phi_{j}\left|\frac{U}{\left|x_{2}-x_{1}\right|}\right| \psi \phi_{j}\right\rangle-\left[\frac{\pi^{2}}{2 \min \left(a_{j}, a_{j-1}\right)^{2}}+\frac{2 \pi^{2}}{L_{j}^{2}}\right]\left\|\psi \varphi_{j}\right\|^{2} .
\end{gathered}
$$

If $\varphi_{j}\left(x_{1}, x_{2}\right) g_{j, u_{1}, u_{2}}\left(x_{1}, x_{2}\right) \neq 0$, then,

$$
\begin{gathered}
S_{j-2} \leq\left|x_{2}-x_{1}\right| \leq S_{j}, \\
\left|u_{2}-u_{1}\right|-2 L_{j} \leq\left|x_{2}-x_{1}\right| \leq\left|u_{2}-u_{1}\right|+2 L_{j} .
\end{gathered}
$$

We would like to have $\left|u_{2}-u_{1}\right|-2 L_{j}>0$, so that the balls are effectively separated; this will occur if $S_{j-2}-4 L_{j}>0$. With this condition, if $\left|u_{2}-u_{1}\right|-2 L_{j} \leq 0, \varphi_{j} g_{j, u_{1}, u_{2}}$ is the zero function on $\mathbb{R}^{6}$.

Given $j$, and $L_{j}$ chosen in the manner described, we will now use the following lemma [7, Lemma 3], 
Lemma 3.3. Let $\phi \in L^{2}\left(\mathbb{R}^{6}\right) \otimes \mathcal{F}$ and supp $\phi \subset B_{1} \times B_{2}$ where supp means support in the coordinates of the electrons, and $B_{1}$ and $B_{2}$ are two disjoint balls of radius $L$, separated $a$ distance $d>0$. Then,

$$
\left\langle\phi\left|H_{0}^{2}\right| \phi\right\rangle \geq 2 E^{1}\|\phi\|^{2}-\left\langle\phi\left|\frac{2 \alpha}{\left|x_{2}-x_{1}\right|}\right| \phi\right\rangle-\frac{16 \alpha L}{\pi^{2} d(d+4 L)}\|\phi\|^{2} .
$$

Using this lemma and the fact that,

$$
\begin{aligned}
& \iint\left\langle\psi \varphi_{j} g_{u_{1}, u_{2}}\left|H_{0}^{2}\right| \psi \varphi_{j} g_{u_{1}, u_{2}}\right\rangle d u_{1} d u_{2} \\
= & \iint_{\left|u_{2}-u_{1}\right|>2 L_{j}}\left\langle\psi \varphi_{j} g_{u_{1}, u_{2}}\left|H_{0}^{2}\right| \psi \varphi_{j} g_{u_{1}, u_{2}}\right\rangle d u_{1} d u_{2},
\end{aligned}
$$

we find that (31) is bounded from below by,

$$
\left[2 E^{1}-\frac{16 \alpha L_{j}}{\pi^{2}\left(S_{j-2}-4 L_{j}\right) S_{j-2}}+\frac{U-2 \alpha}{S_{j}}-\frac{\pi^{2}}{2 \min \left(a_{j}, a_{j-1}\right)^{2}}-\frac{2 \pi^{2}}{L_{j}^{2}}\right]\left\|\psi \varphi_{j}\right\|^{2},
$$

and the sufficient condition for $j \geq 3$ thus becomes,

$$
U \geq 2 \alpha+\frac{16 \alpha L_{j} S_{j}}{\pi^{2}\left(S_{j-2}-4 L_{j}\right) S_{j-2}}+\frac{\pi^{2} S_{j}}{2 \min \left(a_{j}, a_{j-1}\right)^{2}}+\frac{2 \pi^{2}}{L_{j}^{2}} S_{j} .
$$

Introduce the parameters $b_{i}=\alpha a_{i}, Q_{i}=\alpha S_{i}$ and $T_{i}=\alpha L_{i}$. This allows a factorization of $\alpha$. Let us now consider only the cases $j=2$ and $j=3$. We are led to study the minimization of $G \equiv \max \left(G_{1}, G_{2}\right)$, where max denotes the maximum of its arguments, and $G_{1}$ and $G_{2}$ are the following functions:

$$
\begin{gathered}
G_{1}(u, v)=\left[\frac{7}{3}+\frac{\pi^{2}}{2 \min (u, v)^{2}}\right](u+v), \\
G_{2}(u, v, w)=2+\left[\frac{16 L}{\pi^{2} u(u-4 L)}+\frac{\pi^{2}}{2 \min (v, w)^{2}}+\frac{2 \pi^{2}}{L^{2}}\right](u+v+w),
\end{gathered}
$$

where $u, v$ and $w$ are positive parameters. It can be seen that in $G_{2}$ there is no dependence upon $L$ explicitly stated as an argument. This stems from the fact that, given $u>0$, it is always possible to minimize the part of the function that depends on $L$. This we will do now: consider the expression,

$$
\frac{16 L}{\pi^{2} u(u-4 L)}+\frac{2 \pi^{2}}{L^{2}}
$$

and write $L=t u$, with $0<t<1 / 4$, then this function of $L$ becomes,

$$
\frac{4}{\pi^{2} u}\left(\frac{t}{4^{-1}-t}+\frac{\pi^{4}}{2 t^{2} u}\right)
$$

and writing $t=r \pi^{2} / \sqrt{2 u}$, we find,

$$
\frac{4}{\pi^{2} u}\left(\frac{r}{c-r}+\frac{1}{r^{2}}\right)
$$


with $c=\sqrt{2 u} / 4 \pi^{2}$. Introduce now the variable $y=r /(c-r)$, and then, the expression is transformed into,

$$
\frac{4}{\pi^{2} u}\left[y+\frac{1}{c^{2}}+\frac{2}{c^{2} y}+\frac{1}{c^{2} y^{2}}\right]
$$

with $y$ lying in $(0, \infty)$. The positive value of $y$ that minimizes the expression (43) will satisfy,

$$
y^{3}-\frac{2}{c^{2}} y-\frac{2}{c^{2}}=0 .
$$

Depending on the value of $u$, this equation will have three real roots, or one real and two complex. But an upper bound for $u$ is available: recall that we are looking for a minimum value that is smaller than 53.2. Since,

$$
\left[\frac{7}{3}+\frac{\pi^{2}}{2 \min (u, v)^{2}}\right](u+v) \geq \frac{7}{3} u,
$$

we require $u \leq 22.8=3 \times 53.2 / 7$. Therefore, the only positive root will be given by,

$$
\frac{8 \pi^{2}}{\sqrt{3 u}} \cos \left[\frac{1}{3} \arccos \left(\frac{3 \sqrt{3 u}}{8 \pi^{2}}\right)\right] \equiv \frac{8 \pi^{2}}{\sqrt{3 u}} \Gamma(u) .
$$

It may be verified directly this is the only positive solution by using the identity $\cos (3 \theta)=$ $4 \cos ^{3} \theta-3 \cos \theta$. Thus, the original expression has been minimized, and $G_{2}(u, v, w)$ becomes,

$$
G_{2}(u, v, w)=2+\left[\Omega(u)+\frac{\pi^{2}}{2 \min (v, w)^{2}}\right](u+v+w),
$$

where,

$$
\Omega(u) \equiv \frac{32}{\sqrt{3 u^{3}}} \Gamma(u)+\frac{32 \pi^{2}}{u^{2}}+\frac{8}{\Gamma(u)} \sqrt{\frac{3}{u^{3}}}+\frac{3}{2 \pi^{2} u \Gamma(u)^{2}} .
$$

We see that it suffices to study $\max \left(G_{1}, G_{2}\right)$ in the region $0<w \leq v \leq u \leq 22.8$. This is due to two properties: if $\left(u_{0}, v_{0}, w_{0}\right)$ corresponds to a global minimum, then $v_{0} \leq u_{0}$; if $\left(u_{0}, v_{0}, w_{0}\right)$ corresponds to a global minimum in which $v_{0}<w_{0}$, then $G\left(u_{0}, v_{0}, v_{0}\right) \leq G\left(u_{0}, v_{0}, w_{0}\right)$. We have found numerically that for the parameters $u=15.716, w=v=3.9706, \max \left(G_{1}, G_{2}\right)$ is a number smaller than 52.1 .

As for the rest of the conditions $j \geq 4$, first recall that in the notation introduced earlier, $b_{1}=u, b_{2}=v, b_{3}=v$. Putting $b_{j}=(j-2) v$, for $j \geq 4$, we find from equation (37) that the sufficient condition becomes,

$$
\begin{aligned}
U \geq & {\left[2+\frac{16 \beta_{j}}{\pi^{2}\left(1-4 \beta_{j}\right)} \frac{2 u+[2+(j-2)(j-1)] v}{2 u+[2+(j-4)(j-3)] v}\right.} \\
& +\frac{\pi^{2}}{4(j-3)^{2} v^{2}}\{2 u+[2+(j-2)(j-1)] v\} \\
& \left.+\frac{4 \pi^{2}}{\beta_{j}^{2}} \frac{2 u+[2+(j-2)(j-1)] v}{\{2 u+[2+(j-4)(j-3)] v\}^{2}}\right] \alpha
\end{aligned}
$$

where $\beta_{j}$ is some number between 0 and $1 / 4$. 
It can be seen that the sequences,

$$
\begin{gathered}
\frac{(j-2)(j-1)}{2 u+[2+(j-4)(j-3)] v}, \\
\frac{(j-2)(j-1)}{(j-3)^{2}},
\end{gathered}
$$

are monotone decreasing provided that $j \geq 4+u / v$. For the values of $u$ and $v$ already found, it will suffice to take $j \geq 8$. If $\beta_{j}=1 / 4.5$ for $j \geq 4$, all the conditions with $4 \leq j \leq 8$ are smaller than $52.1 \alpha$. Thus, every condition is satisfied if $U$ is larger than or equal to this value. This concludes the proof.

\section{THE CASE OF WEAK COUPLING}

The main result of this section is,

Theorem 4.1. Let $0<\alpha<3 / 4$. Then no binding occurs if,

$$
U \geq 40.4 \alpha+5.94 \alpha^{2}\left(1-\frac{4 \alpha}{3}\right)^{-5 / 2} .
$$

We start with a theorem of Lieb and Yamazaki [18, Equation 24],

Theorem 4.2. Denote by $E^{1}$ the ground-state energy of the polaron. Let $p \geq 2 \alpha / 3$. Then,

$$
E^{1} \geq-\frac{3}{2}\left[1-\left(1-\frac{2 \alpha}{3 p}\right)^{1 / 2}\right]-\frac{1}{2} p \alpha .
$$

Given $\alpha>0$, choose a sufficiently large value for $p$, and set $\beta=-2 \alpha / 3 p$. Note that $-1 \leq \beta<0$. Then the lower bound for $E^{1}$ becomes,

$$
E^{1} \geq-\frac{3}{2}\left[1-(1+\beta)^{1 / 2}\right]+\frac{\alpha^{2}}{3 \beta}
$$

Let $-1<\beta<0$; thus by Taylor Theorem,

$$
E^{1} \geq \frac{\alpha^{2}}{3 \beta}+\frac{3}{4} \beta-\frac{3}{16} \beta^{2}+\frac{3}{32} \beta^{3}(1+\xi)^{-5 / 2},
$$

where $\xi$ is some constant in the interval $(\beta, 0)$.

If the first two terms are minimized with respect to $\beta$, the minimum corresponds to $\beta=$ $-2 \alpha / 3$. This minimum will be attained provided that $\alpha<3 / 2$. Assume henceforth that $\alpha$ satisfies this condition, and then, the following lower bound is obtained,

$$
E^{1} \geq-\alpha-\frac{\alpha^{2}}{12}-\frac{\alpha^{3}}{36}\left(1-\frac{2 \alpha}{3}\right)^{-5 / 2}
$$

Recall what Lemma 1 said. We will improve that lemma in the case that $\alpha$ is sufficiently close to 0:

Lemma 4.3. Let $\phi \in L^{2}\left(\mathbb{R}^{6}\right) \otimes \mathcal{F}$ and $0<\alpha<3 / 4$. Then,

$$
\left\langle\phi\left|H_{0}^{2}\right| \phi\right\rangle \geq\left[2 E^{1}-\frac{4 \alpha^{2}}{3}-\frac{2 \alpha^{3}}{9}\left(1-\frac{4 \alpha}{3}\right)^{-5 / 2}\right]\|\phi\|^{2} .
$$


In [7. Equation 2.26], the following inequality was proved: given $\phi \in L^{2}\left(\mathbb{R}^{6}\right) \otimes \mathcal{F}$ and $\alpha>0$,

$$
\left\langle\phi\left|H_{0}^{2}\right| \phi\right\rangle \geq\left[E^{1}(2 \alpha)-\alpha^{2}\right]\|\phi\|^{2} .
$$

Assuming that $\alpha<3 / 4$, by (56),

$$
\left\langle\phi\left|H_{0}^{2}\right| \phi\right\rangle \geq\left[-2 \alpha-\frac{4 \alpha^{2}}{3}-\frac{2 \alpha^{3}}{9}\left(1-\frac{4 \alpha}{3}\right)^{-5 / 2}\right]\|\phi\|^{2} .
$$

Gurari [13], Lee, Low and Pines [14, 15], and Feynman [6] have derived the following upper bound for $E^{1}$,

$$
E^{1} \leq-\alpha
$$

Valid for all $\alpha>0$. By using this bound on $-2 \alpha$, the result is obtained.

We now proceed in the following way: This lemma will be used in the conditions $j=1$ and $j=2$, the remaining ones being unaltered. As in what was done above, if the condition for $j=2$ is satisfied, $j=1$ will also hold. For $j=2$ the sufficient condition will be,

$$
U \geq\left[\frac{4}{3}+\frac{\pi^{2}}{2 \min \left(b_{1}, b_{2}\right)^{2}}+\frac{2 \alpha}{9}\left(1-\frac{4 \alpha}{3}\right)^{-5 / 2}\right]\left(b_{1}+b_{2}\right) \alpha .
$$

Working as if the third term were absent (in both $j=1$ and $j=2$ ), we are in the same situation as before, except that the first term has been modified from $7 / 3$ in equation (38) to $4 / 3$. As in the previous section, it is found that the minimum of the maximum of the corresponding two functions ( $G_{1}$ with parameter $4 / 3$ and $G_{2}$ without modifications) is smaller than 40.4, choosing the parameters $u=21.41, v=w=5.302$. The rest of the conditions are satisfied by doing the same procedure that was done before: choosing $b_{j}=(j-2) v$, it suffices to study $4 \leq j \leq 9$, and (in the same notation) with $\beta_{j}=1 / 4.5$, it is seen that all the conditions with $j \geq 4$ are smaller than 40.4 . Note that, with $b_{1}$ and $b_{2}$ being appropiate values,

$$
\left[\frac{4}{3}+\frac{\pi^{2}}{2 \min \left(b_{1}, b_{2}\right)^{2}}\right]\left(b_{1}+b_{2}\right) \leq 40.4
$$

and thus if,

$$
U \geq 40.4 \alpha+5.94 \alpha^{2}\left(1-\frac{4 \alpha}{3}\right)^{-5 / 2},
$$

no bipolaron is formed (the addition of the neglected term raises only the conditions $j=1$ and $j=2$ ). This expression is valid for any $\alpha<3 / 4$. One may feel uncomfortable with the fact that the law is no longer linear: it is not of the form $U \geq C \alpha$, with $C$ being a constant; however, to see the practical usefulness of the expression $(63)$, suppose that $\alpha \leq 0.1$. This is not a severe restriction; in fact, many materials satisfy this relation: for example, GaAs, GaSb, InP, InAs and InSb, all have a coupling constant smaller than 0.1 (for instance, the last one mentioned has $\alpha=0.015)$ [3, Table 1]. In this case,

$$
5.94 \alpha\left(1-\frac{4 \alpha}{3}\right)^{-5 / 2} \leq 0.9
$$

and thus for $\alpha \leq 0.1$, no binding occurs if $U \geq 41.3 \alpha$. We could proceed further, and determine the unique value $\alpha_{0}$ such that, for any $\alpha<\alpha_{0}$, this estimate is better than the previous one, $U \geq 52.1 \alpha$. This can be done by solving a fifth degree equation, and we have found that $\alpha_{0}>0.367$. In an analogous way, given $\varepsilon>0$, the unique value $\alpha_{1}>0$ such that if $\alpha \leq \alpha_{1}$, 
then no binding occurs provided $U \geq(40.4+\varepsilon) \alpha$, can be found by solving a similar fifth degree equation.

\section{The CASE OF STRONG COUPLING}

For large enough $\alpha$ we can also obtain an improved bound, though this time we will not be able to determine explicitly a value $\alpha_{2}$ such that for any $\alpha>\alpha_{2}$, the bound is actually better than $U \geq 52.1 \alpha$ :

Theorem 5.1. Let $\varepsilon>0$. Then there is a $\alpha_{2}>0$ such that for any $\alpha \geq \alpha_{2}$, if,

$$
U \geq(38.7+\varepsilon) \alpha
$$

then $E_{U}^{2}=2 E^{1}$.

We start with a bound by Lieb and Thomas [17, Equation 31; see also Erratum]: for all $\alpha>0$

$$
E^{1} \geq-c_{p} \alpha^{2}-o\left(\alpha^{2}\right)
$$

where the term $o\left(\alpha^{2}\right)$ is positive and the notation means that if it is divided by $\alpha^{2}$, it vanishes as $\alpha \rightarrow \infty$. Also, $E^{1} \leq-c_{p} \alpha^{2}$, where, to five decimals of precision, $c_{p}=0.10851$; this is explained in the next chapter, it arises from the Pekar functional. Using these two bounds appropiately, together with equation (58), the following lemma can be proved:

Lemma 5.2. Let $\phi \in L^{2}\left(\mathbb{R}^{6}\right) \otimes \mathcal{F}$ and $\alpha>0$. Then,

$$
\left\langle\phi\left|H_{0}^{2}\right| \phi\right\rangle \geq\left[2 E^{1}-1.218 \alpha^{2}-o\left(\alpha^{2}\right)\right]\|\phi\|^{2} .
$$

The argument is now just as in the previous section: it suffices to study $j \geq 2$, we do as if the term $o\left(\alpha^{2}\right)$ were absent, and compute the minimum of the maximum of $G_{1}$ and $G_{2}$, with $7 / 3$ in $G_{1}$ replaced by 1.218 , and find $U \geq 38.7 \alpha$ as a sufficient condition, with $u=22.52$, $v=w=5.559$. The same method consisting of choosing $b_{j}=(j-2) v$ for $j \geq 4$ is applied, and again all the conditions with $j \geq 4$ are smaller than 38.7. Putting the discarded term only raises $j=1$ and $j=2$, and so the sufficient condition becomes,

$$
U \geq\left[38.7+\frac{o\left(\alpha^{2}\right)}{\alpha^{2}}\right] \alpha .
$$

By definition, for each $\varepsilon>0$, there is a $\alpha_{2}>0$, such that for any $\alpha \geq \alpha_{2}$, no binding occurs if,

$$
U \geq(38.7+\varepsilon) \alpha
$$

\section{Absence of Binding in the Pekar-Tomasevich Bipolaron}

In [7, Section 5.2], the absence of binding in what is known as the Pekar-Tomasevich bipolaron has been studied. We do not wish to give now a full exposition of this model: information about it can be found in the cited reference. We would like only to remark that exactly the same method that was used in Section 3 can be applied here, except that (similarly to what occurred in the previous two sections) $7 / 3$ has to be changed to 0.654 . For all $\alpha$, the condition $U \geq 29.3 \alpha$ is obtained, with parameters $u=31.97, w=v=7.692$. This is quite a modest reduction: the result found in [7] was $U \geq 29.4 \alpha$. 


\section{Acknowledgements}

One of us (RB) would like to thank the School of Mathematics of the Institute for Advanced Study for their hospitality while this work was completed.

\section{REFERENCES}

[1] A. S. Alexandrov, N. Mott, Polarons and bipolarons. World Scientific, Singapore, 1996.

[2] A. Alexandrov and J. Ranninger, Bipolaronic superconductivity, Phys. Rev. B 24, 1164-1169 (1981).

[3] J. Appel, Polarons, in: Solid State Physics, Advances in Research and Applications, Vol. 21, F. Seitz, D. Turnbull, H. Ehrenreich, ed., Academic Press, NY, 1969.

[4] F. Brosens, S. N. Klimin, J. T. Devreese, Variational path-integral treatment of a translation invariant many-polaron system, Phys. Rev. B 71, 214301-214313 (2005).

[5] H. L. Cycon, R. G. Froese, W. Kirsch, B. Simon, Schrödinger operators. Springer Verlag, Berlin, 1987.

[6] R. P. Feynman, Slow electrons in a polar crystal, Phys. Rev. 97, 660-665 (1955).

[7] R. L. Frank, E. H. Lieb, R. Seiringer, L. E. Thomas, Bipolaron and N-polaron binding energies, Phys. Rev. Lett. 104, 210402 (2010).

[8] R. L. Frank, E. H. Lieb, R. Seiringer, L. Thomas, Stability and absence of binding for multi-polaron systems, Publications Mathématiques de L'IHÉS, to appear, published online in February, 2011.

[9] H. Fröhlich, Theory of electrical breakdown in ionic crystals, Proc. R. Soc. Lond. A 160, 230-241 (1937).

[10] H. Fröhlich, Electrons in lattice fields, Adv. in Phys. 3, 325-362 (1954).

[11] M. Griesemer, Exponential decay and ionization thresholds in non-relativistic quantum electrodynamics, J. Funct. Anal. 210, 321-340 (2004).

[12] M. Griesemer, J. S. Møller, Bounds on the minimal energy of translation invariant $N$-polaron systems, Commun. Math. Phys. 297, 283-297 (2010).

[13] M. Gurari, Self-Energy of slow electrons in polar materials, Phil. Mag. Ser. 7 44, 329 - 336 (1953).

[14] T. D. Lee, F. E. Low, D. Pines, The motion of slow electrons in a polar crystal, Phys. Rev. 90, 297-302 (1953).

[15] T. D. Lee, D. Pines, The motion of slow electrons in polar crystals, Phys. Rev. 88, 960-961 (1952).

[16] E. H. Lieb, R. Seiringer, The stability of matter in quantum mechanics. Cambridge University Press, Cambridge, UK, 2010.

[17] E. H. Lieb, L. E. Thomas, Exact ground state energy of the strong-coupling polaron, Commun. Math. Phys 183, 511-519 (1997). Erratum: ibid. 188, 499-500 (1997).

[18] E. H. Lieb, K. Yamazaki, Ground-state energy and effective mass of the polaron, Phys. Rev. 111, 728-733 (1958).

[19] O. Madelung, Introduction to Solid State Theory. Springer-Verlag, Berlin, 1978.

[20] M. A. Smondyrev, V. M. Fomin, Pekar-Fröhlich bipolarons, pp. 13-70, in: Polarons and applications, Proceedings in Nonlinear Science, V.D. Lakhno, ed., John Wiley \& Sons, Chichester, NY, 1994.

[21] G. Verbist, F. M. Peeters, J. T. Devreese, Large bipolarons in two and three dimensions, Phys. Rev. B 43, 2712-2720 (1991).

[22] G. Verbist, M. A. Smondyrev, F. M. Peeters, J. T. Devreese, Strong-coupling analysis of large bipolarons in two and three dimensions, Phys. Rev. B 45, 5262-5269 (1992).

${ }^{1}$ Departmento de Física, P. Universidad Católica de Chile, Casilla 306, Santiago 22, Chile, E-mail address: rbenguri@fis.puc.cl

2 Departmento de Matemática, Facultad de Matemáticas, P. Universidad Católica de Chile, Casilla 306, Santiago 22, Chile,

E-mail address: gabley@uc.cl 